\title{
La relation entre imagination et perception : différence ou répétition?
}

\author{
Par DÉLIA POPA
}

Université catholique de Louvain

Résumé Cette étude revient sur le rapport entre imagination et perception en interrogeant la répétition qui intervient dans l'acte d'imagination. L'analyse de la temporalité propre à l'imagination permet d'observer que cette répétition est solidaire d'un essai toujours renouvelé de rendre présent l'absent. L'hypothèse développée ici est que cette mise en présence opérée par la figurabilité imaginative intervient sur le fond de la perte irrémédiable des contenus réels de notre expérience dans le temps. Fondée dans le souvenir, l'imagination permet de cultiver en rapport avec ces contenus éphémères une forme d'éternité.

\section{Introduction}

Je me propose d'explorer dans ce qui suit la relation entre imagination et perception, avec, pour objectif, l'intention de clarifier le statut de l'acte d'imagination au sein de la sensibilité entendue au sens large. Cette intention de clarification n'est pas absente des textes husserliens qui consacrent à la description des actes imageants des analyses de plus en plus détaillées afin de comprendre leur formation et leur fonction dans la famille plus large des actes intentionnels intuitifs. Tout en distinguant deux formes d'imagination - la phantasia et la conscience d'image -, Husserl en vient alors à parler d'un conflit qu'elles déclarent face à la conscience perceptive, conflit fondé dans la contre-tension (Widerspannung) ${ }^{1}$ coextensive de l'apparition de toute

\footnotetext{
${ }^{1}$ E. Husserl, Phantasia, conscience d'image, souvenir. De la phénoménologie des présentifications intuitives. Textes posthumes (1898-1925), tr. fr. R. Kassis et J.-F.
} 
image $^{1}$ qui fait effraction dans son fond perceptif, ainsi que la possibilité de plonger dans un monde imaginaire qui ne saurait interférer avec le champ couvert par la perception ${ }^{2}$. Lorsque nous imaginons, nous sommes «ailleurs », mais cet «ailleurs » entretient avec le «ici» de la perception des rapports tendus, difficiles.

Bien qu'elle passe le plus souvent inaperçue, cette différence entre imagination et perception scinde le champ de notre expérience intuitive en y introduisant des dénivèlements, en séparant des strates de notre vie sensible qui ne se valent pas, bien qu'elles se recouvrent parfois. La preuve de cette ségrégation est à trouver, selon Husserl, dans le fait que ces deux régimes sensibles de la perception et de l'imagination s'excluent mutuellement. Pour le dire en d'autres termes, entre imagination et perception il y aurait une différence qui fait que les deux ne peuvent coexister sans se nier. Cela se passe comme si l'acte d'imagination était porteur d'une négation à l'égard de l'acte de perception ${ }^{3}$. Mais quelle est la source de cette négation et de ce conflit que l'acte d'imaginer introduit dans la vie sensible?

Cette question se complique si l'on tient compte du fait que l'acte d'imagination est intimement lié à la perception, du moins si l'on suit les descriptions de Husserl, qui ne se contentent pas de souligner la différence entre ces deux types de conscience intuitive, mais exhibent également la composante perceptive que l'on peut trouver le plus souvent dans les actes d'imagination. En effet, si l'on tient compte du fait que la visée d'une image est fondée dans la perception et du fait que la phantasia peut être elle-même perceptive, on peut en déduire qu'il y a finalement peu d'actes d'imagination qui soient non-perceptifs. Rigoureusement parlant, seule la phantasia pure satisfait à cette exigence, mais ses rapports à la perception n'en sont pas moins ambivalents. Il en est ainsi au moins pour deux raisons :

- parce qu'il est très difficile d'isoler des éléments de phantasia qui soient exempts de tout rapport au perçu ;

Pestureau revue par J.-F. Pestureau et M. Richir, Grenoble, Millon, coll. « Krisis », $2002, \mathrm{n}^{\circ} 1, \S 25$, p. 89 (Hua XXIII, p. 51).

${ }^{1}$ C'est ce que Fink a appelé le caractère de fenestrité (Fensterhaftigkeit) de l'image. E. Fink, De la phénoménologie, tr. fr. D. Franck, Paris, Minuit, 1974, p. 92.

${ }^{2}$ « Le champ-de-phantasia entier est en conflit avec le champ perceptif entier, et sans aucune interférence. » E. Husserl, Phantasia, conscience d'image, souvenir, op. cit., $\mathrm{n}^{\circ} 1, \S 32$, p. 102 (Hua XXIII, p. 68).

${ }^{3}$ Cf. R. Bernet, Conscience et existence. Perspectives phénoménologiques, Paris, PUF, coll. «Épiméthée », 2004, p. 83. Voir aussi J.-P. Sartre, L'Imaginaire, Paris, Gallimard, coll. « Idées », 1940, p. 360. 
- parce que ce moi de phantasia par excellence qu'est le moi onirique le sujet rêvant et rêveur — n'est pas un moi qui imagine, mais un moi qui perçoit ${ }^{1}$.

Comment un acte imageant peut-il nier l'acte perceptif sur lequel il est fondé ? Comment le moi onirique peut-il vivre dans un monde séparé du monde de la perception, si lui-même n'imagine pas, mais perçoit? Si l'on s'arrête sur cet aspect de l'acte imageant onirique, on ne peut qu'être saisi par l'impuissance des analyses noétiques à explorer la différence entre imagination et perception. L'impression qui s'en dégage est que plus on avance dans la description de cette différence, plus on est contraint de reconnaître que l'imagination et la perception se chevauchent, empiètent l'une sur l'autre, se mélangent. Pour aller dans la direction que je voudrais donner à ce problème : plus on avance dans la description de la différence entre imagination et perception, plus on a l'impression que ce qui se joue dans l'imagination est une forme de répétition des éléments insignes de la scène perceptive.

J'essayerai de donner corps à cette hypothèse en m'appuyant sur les analyses husserliennes de l'imagination. Mais pour rendre justice à ce qui a constitué la véritable provocation qui est à la source de ce problème, il convient de faire un détour préalable pour remonter en deçà de Husserl, vers Kant, et pour aller au-delà de Husserl, vers Deleuze. Le choix de ces deux références philosophiques n'est pas fortuit, dans la mesure où, d'une part, Husserl reprend dans sa théorie de l'intentionnalité la tripartition des facultés kantiennes ${ }^{2}$, en reformulant, de ses propres aveux, le projet d'une esthétique transcendantale ${ }^{3}$; et dans la mesure où, d'autre part, Deleuze propose luimême une théorie des synthèses passives et de la subjectivité qui présente plusieurs points de convergence avec le projet phénoménologique ${ }^{4}$.

Ce que je vais retenir de Kant, c'est l'idée que l'imagination est à retrouver parmi les trois « sources subjectives de connaissance sur lesquelles

\footnotetext{
${ }^{1}$ «Le Je du monde du rêve ne rêve pas, il perçoit », Lettre de Husserl à Héring, tr. fr. P. Ducat in Alter, «Veille, sommeil, rêve ", n ${ }^{\circ} / 1997$, p. 189.

${ }^{2}$ Cf. J. English, «La Différenciation de l'intentionnalité en ses trois modes canoniques comme problème constitutif central de la phénoménologie transcendantale » in Recherches husserliennes, 1994, vol. 1, pp. 47-72.

${ }^{3}$ E. Husserl, Méditations cartésiennes, tr. G. Peiffer et E. Lévinas, Paris, Vrin, 1986.

4 Pour le rapport de Deleuze à la phénoménologie voir D. Janicaud, $L a$ phénoménologie éclatée, Paris, Éditions de l'Éclat, 1998.
} 
repose la possibilité d'une expérience en général ${ }^{1}$ aux côtés de la sensibilité et de l'entendement. Il s'agit, plus précisément, de trois formes de synthèse : la synthèse d'appréhension qui se joue au niveau des sens (ce que, dans le langage noétique husserlien, on appellera «perception»), la synthèse de reproduction de l'imagination et la synthèse de recognition de l'aperception intellectuelle qui me permet de reconnaître l'objet X comme correspondant à l'aperception pure «je pense ». Ce sont là les coordonnées fondamentales d'un modèle de la pensée qui subordonne la sensibilité à l'entendement, moyennant une opération de l'imagination qui demeure finalement assez mystérieuse. La première édition de la Critique de la raison pure parle d'une synthèse de reproduction par laquelle ce qui a été appréhendé une fois par les sens est repris, répété, reproduit ; mais la deuxième édition l'étayera comme relevant d'un " art caché » qui consiste à concilier ces instances hétérogènes de la subjectivité que sont la sensibilité et l'entendement. La répétition de l'imagination s'avère en effet paradoxale, dans la mesure où son rôle est de capturer la diversité sensible dans l'unité conceptuelle de l'entendement une opération qui ne peut être saisie seulement au niveau empirique où une image peut reproduire une chose vue, mais nécessite le passage par le schématisme transcendantal qui ne reproduit pas, mais produit des schèmes dynamiques par le biais desquelles les formes sensibles sont subsumées aux concepts purs de l'entendement.

Deleuze analysera ce modèle kantien de la pensée comme un paradigme du modèle de la recognition ${ }^{2}$, qui se définit par l'exercice concordant de toutes les facultés, dans un essai de reconnaître toujours dans l'expérience ce qui formellement s'y trouvait déjà, en escamotant toute rencontre possible avec quelque chose de nouveau. Ce modèle est aux yeux de Deleuze hautement problématique non seulement à cause de cette exclusion a priori de toute possibilité de la nouveauté, mais aussi en raison des conditions de cette exclusion, qui sont selon lui celles d'un décalque des structures transcendantales sur des actes empiriques. Deleuze oppose à ce modèle de la pensée un autre modèle : celui qui repose sur une discorde des facultés, sur un " effort divergeant $»^{3}$ qui pousse l'usage de chacune d'entre elles à ses limites. Le point de départ de cette autre scène de la pensée est «une violence originelle faite à la pensée », l'idée que « ce qui est premier dans la

\footnotetext{
${ }^{1}$ E. Kant, Critique de la raison pure, tr. A. Tremesaygues et B. Pacaud, PUF, 1944, p. 129

${ }^{2}$ G. Deleuze, Différence et répétition, Paris, PUF, 1968, p. 174.

${ }^{3}$ Ibid., p. 184.
} 
pensée, c'est l'effraction, la violence ${ }^{1} \gg$ et non pas la tendance à l'unité qui anime l'œuvre de l'entendement. Dans cette perspective, on ne pense pas pour donner une cohérence au champ de notre expérience, mais sous une contrainte qui fait diverger toutes nos tentatives d'unification et de subsomption conceptuelle. Toutefois, le rôle de l'imagination dans ce cadre n'en est pas moins ambigu, car ce qui se joue en elle est bien une forme de répétition. Seulement, cette répétition sera plutôt une espèce de réfraction de cette première effraction sensible qui meut la pensée - et le médium de tout changement possible.

La phénoménologie husserlienne de l'imagination me semble se mouvoir entre les deux modèles de pensée que je viens d'évoquer : proche de la perspective deleuzienne dans son essai de repenser l'esthétique, le rôle fondateur de la perception et la pluralité des modes intentionnels ; proche de Kant par la prééminence de la donation de sens (Sinngebung) intentionnelle qui identifie des unités signifiantes dans la diversité de ce qui est saisi. La fonction qu'elle assigne à l'imagination est toutefois différente de celle que lui assignent ces deux auteurs. Peut-on encore parler ici d'une répétition qui se joue dans l'imagination?

\section{La répétition au sein de l'imagination}

Lorsque Husserl analyse l'imagination dans les Recherches logiques (1901), il la décrit comme un acte intentionnel de présentification (Vergegenwärtigung) qu'il distingue clairement de la perception comprise comme acte intentionnel de présentation (Gegewärtigung). Derrière cette distinction se trouve tout un débat que la phénoménologie naissante de Husserl mène contre les penseurs empiristes anglais (Locke et Hume), qui avaient fait de l'imagination une connaissance de deuxième degré ouvrant la voie de l'abstraction, connaissance où les impressions sensibles premières apparaissent comme affaiblies, quasi-spectrales. Dans cette perspective que Husserl combat, l'imagination incarne précisément une forme de répétition, puisqu'elle n'est rien d'autre que la reprise, en moins clair et moins intense, des sensations. Mais c'est une répétition évanescente, qui n'a pas le pouvoir de rejouer la force première des sensations, se contentant de la refléter faiblement et de la transférer ainsi sur le plan des idées générales.

Husserl a pour sa part au moins deux raisons de critiquer cette façon d'envisager le rôle de l'imagination au sein de la connaissance : tout d'abord,

${ }^{1}$ Ibid., p. 181. 
parce qu'il est fermement convaincu que ce n'est pas de cette façon qu'il faut comprendre la naissance des généralités, la genèse des idées en nous ne provenant pas d'un affaiblissement des impressions sensibles, mais d'une « aperception» (un acte noétique spécifique) qui leur donne un sens objectif; ensuite, parce qu'il cherche à consolider le domaine de l'intuition sensible dans lequel il entend fonder la théorie de la connaissance. Cette consolidation passe par une exaltation du rôle de la perception entendue comme «conscience originaire », mais aussi par la reconnaissance d'une autonomie de l'imagination, comprise comme conscience intentionnelle différente et de la perception et de la signification.

Quelle est la spécificité de l'imagination au sein de la théorie phénoménologique husserlienne ? Comme la perception, elle est un acte sensible, qui se rapporte à un objet sensible : l'image. Mais, contrairement à la perception, ce qu'elle vise n'est pas ce qu'elle peut saisir directement (l'objetimage), mais quelque chose qui s'absente en elle, tout en se donnant indirectement (le sujet-image). C'est en vertu de ce caractère indirect de la saisie imageante que Husserl va la rapprocher d'autres types d'actes indirects, tels le souvenir et l'attente, avec lesquels elle forme la famille des présentifications (Vergegenwärtigungen). Mais tout en opérant ce rapprochement, Husserl insiste sur la particularité de cette forme d'intentionnalité au fonctionnement biaisé, qui présente l'avantage non-négligeable de pouvoir remplacer (pour ainsi dire en tout temps et en tout moment) la perception, lorsque l'objet perçu n'est pas directement saisissable (la perception étant liée à un temps et un moment déterminés, où les conditions de la donation en chair et en os peuvent faire défaut). C'est ainsi que l'imagination change subtilement de statut : bien qu'incapable de présenter son objet « en chair et en os » (leibhaftig) comme la perception, elle présente la qualité insigne de pouvoir nous le donner en absence, comme s'il était présent.

Cette fonction de faire «comme si » de l'imagination s'avère particulièrement intéressante dans deux domaines de la description phénoménologique husserlienne: l'eidétique et l'intersubjectivité. Dans le cadre de l'eidétique phénoménologique, l'imagination mobilise le " faire comme si » pour varier de manière indéfinie la donation possible d'un objet, jusqu'à en saisir l'essence irréductible: sur le fond de tous les changements possibles qu'elle peut imprimer à ce qui est perçu, se dessine alors l'essence invariante de cet objet. Mais en engageant ainsi l'imagination comme opération qui nous fait passer des faits aux essences, Husserl ne semble pas s'intéresser tant à la détermination des invariants en eux-mêmes qu'à l'opération de variation comme telle, qui ouvre l'accès à un courant qui coule parallèlement à la vie effective de la conscience : c'est le courant des possibilités qui ne 
dépendent pas directement des faits, mais s'en dégagent librement ${ }^{1}$. C'est précisément par la liberté qui lui est propre que l'imagination dépasse dans ce domaine la perception ${ }^{2}$, ce qui fait dire à Husserl que la fiction « constitue l'élément vital de la phénoménologie et de toutes les sciences eidétiques » ${ }^{3}$.

Le faire « comme si » de l'imagination intervient de manière plus surprenante dans le cadre de la théorie de l'intersubjectivité husserlienne, lorsque Husserl analyse l'apprésentation propre à la sphère de l' "autre ego $»^{4}$. Jusqu'à un certain point, cela se passe ici comme dans le cas de la perception par esquisses d'une maison ou d'un cube, où certains côtés restent nécessairement invisibles tout en étant co-donnés ensemble avec les côtés visibles. De la même manière, dans la perception d'autrui, sa sphère égoïque demeure cachée, à cette différence près qu'elle ne peut jamais devenir visible, contrairement à la maison dont on peut faire le tour ou du cube que l'on peut retourner. Dans le cas de la perception d'autrui, sa vie consciente, son intériorité ne peut jamais m'être donnée directement, cas auquel je ne pourrais plus distinguer entre ma sphère égoïque et la sphère égoïque d'autrui. Cette incommunicabilité des deux sphères se reflète au niveau spatial par le fait que je suis le "point zéro » de cette relation, en étant perpétuellement « ici », tandis que l'autre se trouve «là-bas ». Mais, malgré la séparation qu'elle instaure, il ne s'agit pas pour autant d'une distance infranchissable, et ceci grâce à l'imagination. En effet, tout en restant « ici », je peux faire « comme si j'étais là-bas »" à la place de l'autre, source d'une analogie de type particulier grâce à laquelle je peux partager avec autrui la constitution du sens d'une expérience qui est commune avant d'être mienne.

Ainsi, tout un ensemble de réalités qui ne peuvent pas, pour des raisons différentes, être perçues directement, peuvent être imaginées, ce qui les maintient dans le circuit d'une expérience sensible dont Husserl a fait le nouveau fondement de la théorie de la connaissance. Cela concerne non seulement le domaine éidétique des possibilités et de ce qui est inaccessible dans la sphère de constitution d'autrui, mais aussi ce qui a été vécu une fois et en rapport à quoi on a perdu la possibilité d'un contact, d'un rapport direct, qui peut librement être réactualisé par l'imagination, revécu de telle manière que son sens puisse être rejoué sur la scène de la conscience.

${ }^{1}$ E. Husserl, Méditations cartésiennes, op. cit., § 25.

${ }^{2} \mathrm{Cf}$. E. Husserl, Idées directrices pour une philosophie et une phénoménologie pure, I, tr. P. Ricœur, Paris, Gallimard, coll. « Tel », 1950, §70.

${ }^{3}$ Ibid., p. 227. Souligné dans le texte.

${ }_{5}^{4}$ E. Husserl, Méditations cartésiennes, op. cit., § 50.

${ }^{5}$ Ibid., $\S 54$, p. 192-193. 
C'est donc le rapport insigne que l'imagination entretient avec l'absence $^{1}$ qui la différencie au sein de nos actes sensibles et au sein de la famille des présentifications. Cette absence est le fond sur lequel son effervescence créative se met en place, mais aussi ce qu'elle a pour tâche de ressusciter, de répéter de telle manière qu'elle nous paraisse en tout point différente de ce qu'elle est : à savoir une présence qui nous comble sur le plan fantasmatique. C'est une véritable conversion de l'absence en présence (de ce qui n'est pas ou n'est plus en quelque chose qui est là pour nous) que la répétition de l'imagination effectue, qui fait que, alors même que le sujet d'une image n'existe pas ou plus (que ce soit un paysage représenté par une peinture ou une personne immortalisée sur un cliché photographique), nous prenons un plaisir manifeste à savourer sa présence dans l'image, l'y retrouvant comme dans une deuxième vie, autrement visible et partageable, qui lui aurait été accordée comme par miracle.

On a eu raison de dire que l'imagination procède d'un manque, que le ressort de son élan est à rechercher dans une forme d'insuffisance quant à ce qui se joue dans la champ de l'expérience; mais on a raison également de souligner la liberté avec laquelle cette insuffisance est saisie, de manière à être retournée en profusion fantastique. La richesse de notre imaginaire repose donc sur des bases qui sont des plus précaires, liées à l'absence, au manque et - devrait-on ajouter — à la perte. Car, même si Husserl ne semble pas accorder d'importance à cet aspect, c'est l'expérience temporelle de la perte que l'imagination semble le mieux venir convertir en expérience porteuse de nouvelles possibilités. Si l'on accorde à cet aspect toute l'attention qui lui est due, il apparaît ainsi que ce qui est en jeu dans le renouvellement de l'expérience sensible que l'imagination opère, est une réaction à la perte irrémédiable que nous subissons dans le temps de tout ce qui a été une fois présent pour nous. Pour approfondir le sens de cette remarque, il convient de faire appel aux analyses husserliennes de la temporalité.

\section{Vivre et revivre le temps}

À toute tentative de faire de la perte un phénomène paradigmatique de notre expérience temporelle, on pourrait objecter que le temps n'est pas uniquement un flux où les instants présents s'abîment et disparaissent, mais aussi un

\footnotetext{
${ }^{1}$ La «phénoménalisation de l'absence » a été désignée par R. Bernet comme la « question cruciale de la phénoménologie ». Voir R. Bernet, La vie du sujet, Paris, PUF, 1994, p. 216.
} 
flux à travers lequel des sens se sédimentent, qui peuvent être par la suite réactivés. Afin de cerner ce qui se joue dans la répétition de l'imagination, il convient d'examiner de plus près ce qui est véritablement perdu et ce qui est sédimenté à travers le temps. On constate alors que ce qui en vient à manquer n'est pas la forme du présent comme telle, qui recommence pour ainsi dire automatiquement, nous rattachant de façon indélébile à une impression originaire ${ }^{1}$ infatigable et nous soumettant à ce que Rudolf Bernet a appelé « la dure loi du maintenant qui se renouvelle sans cesse» ${ }^{2}$. Ce qui est perdu relève plutôt de ce que le présent rend possible, de ce avec quoi il nous met en relation directe, qui ne saurait être maintenu comme étant le même à travers le temps : les sens constitués qui passent nécessairement par une phase de déconstitution, des sens institués qui doivent être destitués avant de pouvoir être redécouverts, réactivés.

Tel est le drame de la vie consciente : celui de rester perpétuellement arrimée au présent, tout en assistant à la perte progressive de ce que ce présent rend possible (en termes de contact, de rencontres, d'attachements); de contempler depuis le vertige toujours actif de son «maintenant» ce que ce maintenant ne peut plus contenir, dont il ne reste que des ruines, des traces, des échos et des vestiges, de ce qui est temporairement ou définitivement perdu. C'est peut-être la raison pour laquelle le présent ne marque pas seulement pour nous l'expérience joyeuse d'une renaissance, mais aussi une expérience douloureuse : celle d'être les contemporains d'une impossibilité de rendre contemporains les contenus d'expérience auprès desquels nous aimerions nous trouver. Ce qui fait que parfois nous préférerions être moins présents, pour rejoindre ce que nous avons perdu plutôt que de demeurer dans un présent où il manque.

L'imagination nous offre pour sa part, une autre possibilité : celle de rendre présent ce qui est absent, d'intégrer dans une forme d'intuition directe ce qui désormais ne se donne qu'indirectement, via le souvenir ou l'attente d'une retrouvaille. Husserl a peu analysé le rapport de l'imagination à l'attente ${ }^{3}$. Mais il s'est penché sur les affinités manifestes de l'imagination et du souvenir. En effet, l'imagination partage avec le souvenir le fait de

\footnotetext{
${ }^{1}$ E. Husserl, Leçons pour une phénoménologie de la conscience intime du temps, $\mathrm{tr}$. fr. H. Dussort, Paris, P.U.F., coll. « Épiméthée », 1996, § 31.

${ }^{2}$ R. Bernet, La vie du sujet, op. cit., p. 307.

${ }^{3}$ Pour cette question, voir notre étude «Attendre, prévoir, espérer : modalités de la présomption intentionnelle » in J.-F. Lavigne, J. Leclercq, Espérer - neuf études de phénoménologie, 2014, Louvain-la-Neuve, Presses Universitaires de Louvain, pp. 75-87.
} 
reproduire des éléments qui ne sont pas présents. Ensemble, ils composent une conscience temporelle spécifique que Husserl appelle dans ses Leçons sur la conscience intime du temps la conscience reproductive ou représentative $^{1}$, pour la distinguer de la conscience du présent qui comprend le maintenant (Jetzt) accompagné de tout un horizon de protentions et de rétentions. Ce qui vient juste de se passer et ce qui est sur le point d'advenir ne font pas partie de cette conscience temporelle seconde où le présent est reproduit, où les fantasmes remplacent les sensations ${ }^{2}$. Pour que cette deuxième conscience temporelle formée par le souvenir et l'imagination puisse s'instaurer, un gap doit s'introduire entre elle et la conscience du présent: ce gap est celui de l'oubli — forme la plus élémentaire de la perte - , qui relègue dans les tréfonds de la conscience des contenus vivants qui sont ainsi sédimentés et qui pourront ensuite être réactivés de manière volontaire ou involontaire.

Mais l'imagination ne se confond pas pour autant totalement avec le souvenir : elle semble plutôt s'y fonder ${ }^{3}$, afin de développer un rapport à la perte qui est légèrement différent. Mon hypothèse est que, face à la perte irrémédiable des contenus réels de notre expérience dans le temps, l'imagination permet de cultiver une forme d'éternité. Plus précisément, elle permet de retenir dans un présent qui ne passe plus (qui se conserve) ce qui autrement fait l'objet de la perte : on parle ainsi de l'immortalité des œuvres d'art ou du fait d'immortaliser sur une photographie un moment vécu, dont on sait qu'il ne sera plus, mais qui pourra ainsi demeurer dans la conscience imageante comme quelque chose d'éternellement contemporain, malgré sa disparition. C'est cette ouverture de la dimension de l'éternité qui distingue l'imagination du souvenir, qui ne peut pour sa part que ramener ponctuellement tel ou tel moment vécu, mais en tant que moment passé, sans pouvoir véritablement le retenir et lui conférer une nouvelle forme de présence, sans en faire l'objet d'une nouvelle intuition et y déceler de nouvelles possibilités. Lorsqu'une véritable mise en présence de l'absent est effectuée, c'est le signe que l'imagination a rejoint et surplombé le souvenir ; ce qui y est en jeu n'est plus dès lors une simple visite dans le « musée du passé », mais une nouvelle expérience où la perte du passé est convertie en gain de nouvelles possibilités, en ouverture de nouveaux horizons.

\footnotetext{
${ }^{1}$ E. Husserl, Leçons pour une phénoménologie de la conscience intime du temps, op. cit., § 19, pp. 62-65.

${ }^{2}$ Ibid., Supplément II.

${ }^{3}$ E. Husserl, Idées directrices, op. cit., § 112.
} 
L'éternité de la vie imaginaire est à comprendre comme ce qui est rendu possible par une forme bien particulière de répétition. Car c'est une répétition qui va de pair avec une modification qu'elle imprime aux contenus de conscience, qui fait qu'ils ne reviennent jamais en imagination comme étant exactement les mêmes, mais transformés, pourvus de qualités qu'ils n'avaient pas ou n'avaient pas encore manifestées ou bien placés dans des situations qui leur étaient étrangères. Telle est la force propre à la figuration imaginaire (Verbildlichung) : elle réside en une forme d'élaboration de ce qui a été une fois présent qui le ramène comme disponible, mais qui en même temps l'adapte aux besoins des nouvelles situations où il est revécu en tant qu'imaginé.

\section{Imagination et mémoire : la question de l'habitude}

Parmi les commentateurs de Husserl, celui qui est allé peut-être le plus loin dans l'analyse de la division de la conscience temporelle est Paul Ricœur ${ }^{1}$. En s'attardant sur la complicité du souvenir et de l'imagination, comme relevant de cette conscience temporelle seconde qui n'est accessible que pardelà l'oubli, Ricœur pose la question de la rupture par laquelle elle s'instaure en rapport avec la conscience du présent. Cette rupture n'est nulle autre que celle qui sous-tend le rapport entre imagination et perception. En poussant plus loin les réflexions de Ricœur, on pourrait dire qu'entre les deux consciences, il n'y a pas que différence, mais aussi différend, dans la mesure où s'y dispute le point de gravité de la constitution de sens de l'expérience. Ce sens de l'expérience est-il engendré dans le présent ou bien dans la conscience «seconde » du souvenir et de l'imagination ? ${ }^{2}$ Est-ce en visant directement des contenus d'expérience ou bien en les répétant que l'on produit du sens?

Si l'on veut éviter le choix sans retour devant lequel cette question nous place ${ }^{3}$, la seule solution - husserlienne et ricœurienne - est de persé-

\footnotetext{
${ }^{1}$ P. Ricœur, Temps et récit III. Le temps raconté, Paris, Seuil, 1985, pp. 43-82.

${ }^{2}$ Cette question a été élaborée par J. Derrida et par M. Richir. Derrida insiste sur l'importance de la trace temporelle comme origine du temps, tandis que Richir formule la thèse d'une fondation de la temporalité dans la phantasia.

${ }^{3}$ Il serait trop facile, suivant une lecture rapide de $\mathrm{J}$. Derrida, de dire que, là où pour Derrida le sens s'engendre dans le passé, pour Husserl il serait toujours arrimé à une conscience du présent perpétuellement ingénue et première. Ce qui intéresse Husserl, c'est de rendre compte de la façon dont se tisse l'unité d'une expérience, avec son
} 
vérer dans la recherche d'un principe de continuité entre les deux types de conscience. Husserl le trouvera dans l'entrelacement entre intentionnalité longitudinale et intentionnalité transversale, qui lui permettra de remonter vers une conscience temporelle directement abreuvée par l'impression originaire, qui vise le courant temporel afin de le constituer comme unitaire ${ }^{1}$. Ricœur insistera quant à lui sur l'opération de « recouvrement» (Deckung) par laquelle la conscience reproductive - souvenir et imagination confondus - rejoue ce qui se passe dans le présent ${ }^{2}$. Mais, si l'on regarde de plus près, cette opération n'est pas dépourvue d'ambiguité. Tout d'abord, parce que, comme le terme l'indique, recouvrir n'est pas revivre: entre les deux consciences temporelles il y a des glissements, des omissions et des altérations qui rendent tout rapport entre temps perdu et temps retrouvé difficile, problématique. Ensuite, parce que souvenir et imagination ne se rapportent pas de la même façon à ce qui est perdu dans le présent. Le souvenir prétend à la vérité $^{3}$, il cherche à atteindre dans le passé ce noyau inaltérable qui le ramène à nous tel qu'il a eu lieu, tandis que l'imagination le vise librement, à travers une modification qui travaille - pourrait-on avancer - en écho à la modification qui affecte insidieusement tout contenu temporel présent.

Cette différence qui se creuse dans la conscience reproductive doit être prise au sérieux, dans la mesure où elle fait valoir d'une part une conscience qui s'unifie autour d'un présent auquel les souvenirs sont nécessairement ramenés et d'autre part une conscience qui s' "éparpille » et qui semble décentrer le présent vers un certain passé. Que ce passé ne soit pas le passé vécu tel qu'il a été vécu mais un passé qui a rejoint le cercle somme toute assez sélectif de l'éternité, c'est ce que nous voudrions souligner, afin de saisir dans la répétition de l'imagination une mutation des sens vécus sur une autre scène, qui n'est plus régie par le présent de la conscience subjective. Ce point semble pouvoir être reconnu directement à partir des analyses husserliennes lorsqu'elles insistent sur le caractère passif et réceptif de la conscience reproductive ${ }^{4}$. Comme Ricœur l'a montré, Husserl aboutit par là à un arrimage de la conscience impressionnelle première, désormais omniprésente, à la conscience d'une temporalité objective, composée de tempo-

système de synthèses d'association et d'identification, avec ses concordances qui ne cessent d'opérer par-delà les ratages et les « biffages » de l'intentionnalité.

${ }^{1}$ Pour cette question, voir R. Bernet, La vie du sujet, op. cit., pp. 196-200.

${ }^{2}$ Ce qui permet de parler des rétentions et des protentions qui accompagnent le souvenir. Cf. Temps et récit III, op.cit., p. 68 sq.

${ }^{3}$ Ibid., pp. 67-68.

${ }^{4}$ Cf. Ibid., p. 80-82. 
objets (Zeitobjekte) repérables et de « situations temporelles » inaltérables et donc répétables comme telles.

Rien de moins sûr, cependant, que la possibilité de cet arrimage, sans que le mouvement de l'impression ne soit recouvert par la fixité des instants objectivés, sans que la vivacité de l'impression ne se perde dans les échos monocordes au sein desquels elle est accueillie : c'est le sens premier du recouvrement (Deckung) en tant que transposition (Ubertragung) ou encore en tant que substruction (Substruktion) ${ }^{1}$. En revanche, il y a lieu de montrer, à partir des textes husserliens plus tardifs, que la vivacité de l'impression est soutenue par une multitude de synthèses passives qui s'entretissent et se répondent. C'est à ce niveau qu'il convient de reposer la question de la répétition de l'imagination. Cette question est à examiner dans le cadre husserlien à partir de l'opération de réactivation (Reaktivierung) ${ }^{2}$, mais elle trouve également un traitement inédit chez Deleuze, qui, en suivant Hume, voit dans l'imagination «un pouvoir de contraction $»^{3}$ qui fonde cette synthèse passive de type spécial qu'est l'habitude. Ce qui est spécifique à l'habitude est qu'elle rattache des moments semblables tout en retenant leur différence, qu'elle associe sans niveler et répète sans transposer ${ }^{4}$.

Dans l'habitude, pas de surplomb ou d'occultation: les moments qu'elle contracte sont égaux et leur synthèse d'association toujours renouvelée. C'est la raison pour laquelle l'habitude ne désigne pas, à proprement parler, la fixité objectivable des rituels, mais la vivacité qui les anime, qui

\footnotetext{
${ }^{1}$ Voir Philosophie de l'arithmétique, tr. fr. pp. 169-172 et p. 223. Dans ses notes sur la traduction de Übertragung, J. English définit ainsi le terme de «transposition» : «Il désigne le mécanisme par lequel, une fois que le processus abstractif en se déplaçant est passé d'une première étape à une seconde, il se produit par contrecoup, en sens indirect, une transposition des résultats atteints à la seconde étape sur l'espace occupé par la première, qui du fait même devient incompréhensible, puisqu'on ne peut plus reconnaître le sens qu'elle a commencé par avoir. Il s'agit donc d'une transposition (...) au niveau intrinsèque, dans le sens très précis d'une interversion des moments successifs de la fondation, ce qui se situe après se projetant sur ce qui se situe avant pour le recouvrir et en masquer le sens originaire, au profit d'un autre sens, atteint plus tardivement. » E. Husserl, Philosophie de l'arithmétique, op. cit., p. 415, souligné dans le texte.

${ }^{2}$ Voir notre étude «À la recherche du commencement perdu : la question en retour » in G. Jean et Y. Mayzaud (éd.), Derrière le commencement. Phénoménologie, métaphysique et théologie, Éditions Universitaires Européennes, Sarrebruck, 2012, pp. 25-37.

${ }^{3}$ G. Deleuze, Différence et répétition, op. cit., p. 96.

${ }^{4}$ Ravaisson définit déjà l'habitude comme la contraction d'un mouvement. Voir F. Ravaisson, De l'habitude, Paris, Allia, 2007, p. 7.
} 
témoigne des efforts subjectifs à l'œuvre pour intégrer des ordres sociaux, normatifs, cosmiques. C'est également la raison pour laquelle nos habitudes évoluent, disparaissent et sont instaurées en lien étroit avec les modes de vie que nous adoptons ou inventons. L'habitude cristallise les efforts que nous déployons pour nous accommoder de notre monde - aussi peu accommodant et oppressant qu'il soit —, les portant au-delà d'eux-mêmes, dans la sphère des gestes qui persistent en vertu de leur itération continuelle.

Dire que l'imagination fonde l'habitude, c'est insister sur la fraîcheur et la vivacité dont toute habitude est porteuse, pour autant qu'elle est envisagée phénoménologiquement de l'intérieur de sa pratique et non pas à distance, comme rituel objectivé. C'est également réserver à l'imagination le rôle de « soutirer à la répétition quelque chose de nouveau » ${ }^{1}$, ce qui revient à dériver du pouvoir contractant de l'imagination la force de nous orienter parmi les points de vue multiples selon lesquels on peut saisir une situation vers le point de vue unique qui engage notre pratique effective - ce point de vue où nul ne pourrait nous remplacer - , vers cette perspective où les choses les plus régulières, tel le battement d'un cœur, apparaissent comme portées par des passions singulières, guettées par des discontinuités et des arrêts. Deleuze peut ainsi affirmer que «la répétition dans son essence est imaginaire » et que « la vraie répétition est de l'imagination » ${ }^{2}$ uniquement parce que « la répétition renvoie à une puissance singulière $»^{3}$, à une force de différenciation qui n'est nulle autre que la subjectivité comprise comme mouvement de genèse de toutes les modifications qui nous affectent.

Sur le terrain de la phénoménologie husserlienne, cette description de la répétition de l'imagination comme pouvoir de contraction revient à comprendre l'acte d'imagination comme fondé dans une synthèse passive d'association responsable de l'engendrement de la nouveauté. Son incidence est à examiner sur les deux plans de la genèse du sens et de la genèse de la subjectivité ${ }^{4}$. Mais sens et sujet ne peuvent révéler leur ancrage dans la répétition imageante que dans la mesure où l'acte d'imagination lui-même touche à ses limites et affronte son défi le plus redoutable qu'est l'inimaginable. Ainsi peut-il participer à ce que Deleuze a dépeint comme une dis-

\footnotetext{
${ }^{1}$ G. Deleuze, Différence et répétition, op. cit., p. 103.

${ }^{2}$ Ibid.

${ }^{3}$ Ibid., p. 10.

${ }^{4}$ Pour cette analyse voir notre étude « La temporalité de l'imagination : le projeté et l'imprévisible » in A. Dufourcq (éd.), Est-ce réel ? Phénoménologies de l'imaginaire, Leiden-Boston, Brill, 2016, pp. 42-56.
} 
corde des facultés comme source de «ce qui nous fait penser». Dans ce cadre,

Il faut porter chaque faculté au point extrême de son dérèglement, où elle est comme la proie d'une triple violence, violence de ce qui la force à s'exercer, de ce qu'elle est forcée de saisir et qu'elle est seule à pouvoir saisir (...). Chaque faculté découvre alors la passion qui lui est propre $(. . .)^{1}$.

Comprendre l'acte d'imaginer en rapport avec les autres actes subjectifs, de manière à susciter un exercice vivant de la pensée, reviendrait alors à remonter vers la passion propre de l'imagination, révélée dans ce qui nous pousse à imaginer, dans ce que nous découvrons lorsque nous imaginons et dans ce que nous ne pouvons expérimenter qu'en imaginant, pris dans une répétition qui ne gomme pas la différence, mais la soustrait au milieu des pratiques les plus redondantes, la maintient et s'y consacre.

\section{Bibliographie}

Bernet R., Conscience et existence. Perspectives phénoménologiques, Paris, PUF, coll. « Épiméthée », 2004, p. 83.

Bernet R., La vie du sujet, Paris, PUF, coll. « Épiméthée », 1994.

Deleuze G., Différence et répétition, Paris, PUF, coll. «Épiméthée », 1968, p. 174.

English J., «La Différenciation de l'intentionnalité en ses trois modes canoniques comme problème constitutif central de la phénoménologie transcendantale », Recherches husserliennes, 1994, vol. 1, p. 47-72.

Fink E., De la phénoménologie, tr. fr. D. Franck, Paris, Minuit, 1974, p. 92.

Husserl E., Idées directrices pour une philosophie et une phénoménologie pure, I, tr. fr. P. Ricœur, Paris, Gallimard, coll. « Tel », 1950.

Husserl E., Leçons pour une phénoménologie de la conscience intime du temps, tr. fr. H. Dussort, Paris, PUF, coll. « Épiméthée », 1996, § 31.

Husserl E., Phantasia, conscience d'image, souvenir. De la phénoménologie des présentifications intuitives. Textes posthumes (1898-1925), tr. fr. R. Kassis et J.F. Pestureau revue par J.-F. Pestureau et M. Richir, Grenoble, Millon, coll. « Krisis », 2002.

Husserl E., Lettre de Husserl à Héring, tr. fr. P. Ducat, Alter, «Veille, sommeil, rêve $», \mathrm{n}^{\circ} 5 / 1997$.

Husserl E., Méditations cartésiennes, tr. fr. G. Peiffer et E. Lévinas, Paris, Vrin, 1986.

${ }^{1}$ G. Deleuze, Différence et répétition, op. cit., p. 186. 
Janicaud D., La phénoménologie éclatée, Paris, Éditions de l'Éclat, 1998.

Kant E., Critique de la raison pure, tr. fr. A. Tremesaygues et B. Pacaud, PUF, 1944.

Popa D., "À la recherche du commencement perdu: la question en retour », in

G. Jean et Y. Mayzaud (éds.), Derrière le commencement. Phénoménologie, métaphysique et théologie, Éditions Universitaires Européennes, Sarrebruck, 2012, p. 25-37.

Popa D., «Attendre, prévoir, espérer : modalités de la présomption intentionnelle », in J.-F. Lavigne et J. Leclercq (éds.), Espérer - neuf études de phénoménologie, Louvain-la-Neuve, Presses Universitaires de Louvain, 2014, p. 25-37.

Popa D., «La temporalité de l'imagination: le projeté et l'imprévisible» in

A. Dufourcq (éd.), Est-ce réel? Phénoménologies de l’imaginaire, LeidenBoston, Brill, 2016, p. 42-56.

Ravaisson F., De l'habitude, Paris, Allia, 2007.

Ricœur P., Temps et récit III. Le temps raconté, Paris, Seuil, 1985.

Sartre J.-P., L'Imaginaire, Paris, Gallimard, coll. « Idées », 1940. 